\title{
Severe falciparum malaria with hypophosphataemia and presumed hypophosphatemia induced rhabdomyolysis: a case report
}

\author{
M Perera, V Samarawickrama, K Lokuketagoda, \\ $\mathrm{K}$ Thirumavalavan \\ Sri Lankan Journal of Infectious Diseases 2018 Vol.8 (2):142-146 \\ DOI: http://dx.doi.org/ 10.4038/sljid.v8i2.8225
}

\begin{abstract}
Malaria is eliminated from Sri Lanka, yet cases contracted overseas are still encountered in the country. It is therefore important to be vigilant of possible complications of severe malaria. We report a 31-year-old sailor (Sri Lankan national) with a recent travel history to West Africa who presented with an acute febrile illness of seven days duration. He had high fever, myalgia, drowsiness as well as profuse watery diarrhea. Clinical examination revealed a febrile patient with GCS 15 with no features of meningism. He was icteric with right hypochondrial tenderness.

Investigations showed hemolysis. Microscopy and rapid diagnostic assays revealed Plasmodium falciparum infection. He was started on IV artesunate followed by artemisinin based combination therapy orally to which he responded. On day 4 he developed evidence of rhabdomyolysis with low serum phosphate and normal urine phosphate levels. Hypophosphatemia was considered to be the driving factor for rhabdomyolysis, and phosphate replacement was done for which the patient responded and was discharged on day 10 fully recovered.
\end{abstract}

Key words: Malaria, Plasmodium falciparum, Rhabdomyolysis, Hypophosphatemia

\section{Introduction}

Sri Lanka was certified as having eliminated malaria in 2016 by the WHO. ${ }^{1}$ However, imported cases of malaria are still seen in the country. Severe malaria caused by Plasmodium falciparum is a serious condition with several possible complications. Our case draws attention to a rare complication and its possible etiology.

\section{Case Report:}

A 31-year-old previously healthy male (Sri Lankan national) presented to the casualty unit of the National Hospital, Sri Lanka with an acute febrile illness of seven days

\footnotetext{
${ }^{1}$ National Hospital of Sri Lanka, Sri Lanka Address for correspondence: Dr.HMM Perera, National Hospital of Sri Lanka, Sri Lanka, Tel: +94710658890 Email: manitha321@gmail.com (D) http://orcid.org/0000-0002-9349-741x

Received 01 August 2018 and revised version accepted 18 October 2018 
duration. He is a sailor by profession and gave a history of recent travel to Europe as well as to West Africa in the past few weeks, with return to Sri Lanka two weeks previously. He had high fever of $39.8^{\circ} \mathrm{C}$ with associated chills and rigors. There was no periodicity of fever. There was associated myalgia and malaise. Two days prior to admission, the patient developed profuse watery diarrhea with frequency of 10 times per day. He complained of headache and was drowsy with GCS of 15. There was no photophobia or seizures. He also complained of mild right hypochondrial pain.

On examination, he was febrile with a temperature of $39.8^{\circ} \mathrm{C}$ and was drowsy with GCS of $15 / 15$. There was no neck stiffness and Kernig's sign was negative. He was clinically pale and there was a tinge of icterus. His vital signs were unstable with a pulse rate of $108 / \mathrm{min}$ and blood pressure of $90 / 60 \mathrm{mmHg}$ on admission. His peripheries were warm. Lungs were clear to auscultation. The abdomen was soft with mild hepatomegaly and right hypochondrial tenderness. There was no splenomegaly. The patient was catheterized on admission and was producing adequate normal colored urine.

Investigations revealed the following. Complete blood count WBC $9.24 \times 103 / \mathrm{uL}$ (neutrophil $68.8 \%$ and lymphocytes of $22.9 \%$ ), HB $9.7 \mathrm{~g} / \mathrm{dl}$ and platelets of 27,000/1. The clotting profile was normal. The AST and ALT were $129 \mathrm{U} / \mathrm{L}$ and $79 \mathrm{U} / \mathrm{L}$ respectively with evidence of hyperbilirubinemia at $93 \mu \mathrm{mol} / \mathrm{L}$ with elevation of the indirect fraction. Haemolysis was further supported by an increased LDH at $2385 \mathrm{u} / \mathrm{L}$. The serum creatinine was $146 \mu \mathrm{mol} / \mathrm{L}$, potassium $5.7 \mathrm{mmol} / \mathrm{l}$ and sodium $138 \mathrm{mmol} / \mathrm{l}$. Serum ionized calcium was $1.18 \mathrm{mmol} / \mathrm{L}(1.12-1.32 \mathrm{mmol} / \mathrm{L})$ and serum phosphate was $2.9 \mathrm{mg} / \mathrm{dl}(2.7-4.5 \mathrm{mg} / \mathrm{dl})$. CBS was normal. Ultrasound scan of the abdomen showed mild hepatomegaly with a prominent spleen. Stool full report revealed 30-40 pus cells /HPF with no red cells and negative amoebae, ova or cysts. UFR was normal. Blood picture showed haemolysis with severe thrombocytopenia and neutrophil toxic changes. There were no schistocytes. Microscopy was carefully assessed for malaria due to travel history and the clinical presentation and showed numerous malarial parasites in ring stages of falciparum malaria, schizonts and gametocytes. Subsequent quantification by the anti malarial campaign demonstrated a parasite density of $>500000 / \mu \mathrm{L}$. The malaria rapid diagnostic test also became positive for Plasmodium falciparum. Due to myalgia and acute kidney injury, CPK was done which was normal. Arterial blood gas analysis revealed metabolic acidosis with $\mathrm{pH} 7.31$ with lactate of $6 \mathrm{mmol} / \mathrm{L}$.

Severe falciparum malaria was diagnosed, and patient was started on intravenous artesunate $2.4 \mathrm{mg} / \mathrm{kg} 3$ doses 12 hours apart (0, $12 \mathrm{hrs}$. and $24 \mathrm{hrs}$.) and daily thereafter for 3 days according to the national treatment policy for management of falciparum malaria. IV fluid replacement was done, and supportive transfusion was given. Parasite load gradually declined to $218000 / \mu \mathrm{L}$ by the end of day 2 of treatment and $1000 / \mu \mathrm{L}$ by end of day 3 . The patient's clinical condition was improving gradually with improved sensorium and settling fever and he was switched to a full course of oral Artemisinin based combination therapy. His creatinine rose to a maximum of $224 \mu \mathrm{mol} / \mathrm{l}$ on day 2 . He maintained an adequate urine output and there was no indication for hemodialysis. 
On day 4 in hospital, when the parasite count was almost undetectable, repeat CPK was found to be elevated at $18932 \mathrm{u} / \mathrm{L}$. At this point serum ionized calcium was low $(1.00 \mathrm{mmol} / \mathrm{l})$ and surprisingly, the serum phosphate was also found to be low at 2.0 $\mathrm{mg} / \mathrm{dl}(2.7-4.5 \mathrm{~g} / \mathrm{dl})$. Urinary phosphate remained normal despite hypophosphatemia. Over the ensuing days CPK rose to maximum of $115278 \mathrm{u} / \mathrm{L}$. The creatinine did not rise further. At the height of rhabdomyolysis serum phosphate remained at the lower limit of normal. Usually it is expected to have hyperphosphatemia at the peak of rhabdomyolysis. Persistence of hypophosphatemia in the phase of severe rhabdomyolysis led to possibility of hypophosphatemia as the possible mechanism driving rhabdomyolysis, which led to careful replacement of phosphate. With phosphate replacement rhabdomyolysis gradually improved and settled. During this period patient received alkaline diuresis as a temporizing measure.

By day 10, the patient was fully recovered, and rhabdomyolysis had completely settled and he was discharged after a single dose of primaquine.

The timeline of the patient's clinical course is given below

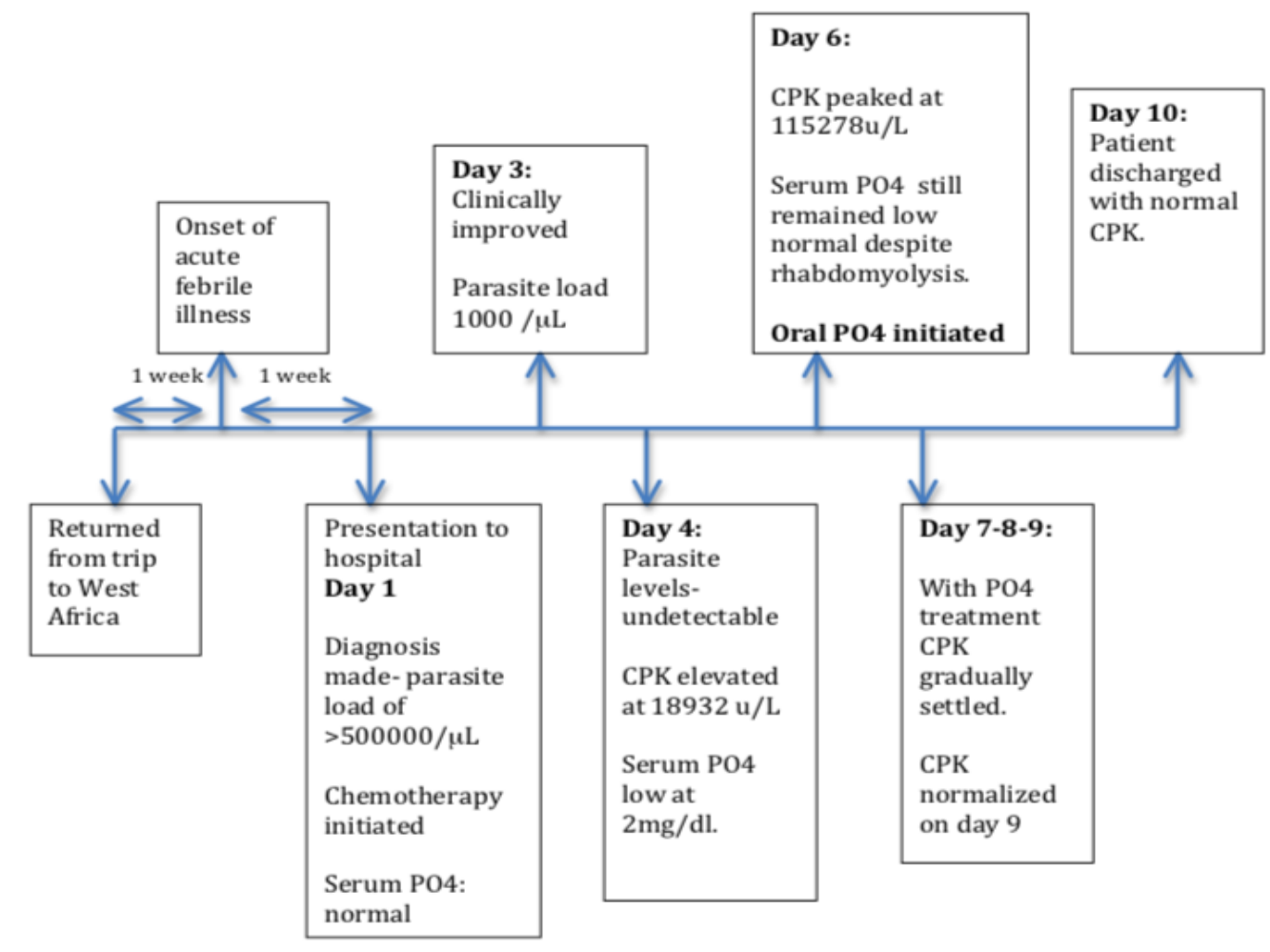

\section{Discussion:}

Severe falciparum malaria is associated with a myriad of serious complications such as cerebral malaria, hypoglycemia, acute renal failure and metabolic acidosis. Rhabdomyolysis is also a recognized yet uncommon complication of severe malaria. There are only handful of cases worldwide and only one reported case in Sri Lanka. ${ }^{2}$ Rhabdomyolysis in severe malaria is thought to be due to several possible mechanisms. $P$. falciparum induces myositis by macrophages and T cells. ${ }^{3}$ It is also postulated that sequestration of red cells may induce microcirculatory occlusion 
leading to muscle damage. ${ }^{4}$ Another mechanism is muscle damage via high TNFalpha levels in malaria. ${ }^{5}$

Electrolyte abnormalities are common in severe malaria. Hypophosphatemia is a recognized manifestation. Several mechanisms are responsible for this. It is assumed that serum phosphate relates inversely with body temperature such that high fever is a cause of hypophosphatemia in malaria. ${ }^{6}$ However a lowered renal threshold for phosphate appears to be a contributing factor too. In our case, presence of normal urinary phosphate excretion in the presence of hypophosphatemia may point towards renal wasting.

Rhabdomyolysis in our patient occurred quite late in the illness when the parasitic burden was undetectable. Therefore, rhabdomyolysis due to high parasitic burden or sequestration of parasitized RBC seemed less likely. As a result, mechanisms other than ones described above were sought. There was no previous alcoholism or poor nutritional state to suggest chronic hypophosphatemia. Usually rhabdomyolysis should result in hyperphosphatemia. Interestingly, in our patient, the serum phosphate remained low despite rhabdomyolysis and remained low normal at the height of rhabdomyolysis. This paved the way to consider hypophosphatemia as the driving factor for rhabdomyolysis.

As a result, the decision was made to carefully replace phosphate, and oral phosphate was started. This was done with careful measurement of serum phosphate to avoid hyperphosphatemia. The patient was also started on alkaline diuresis as a temporizing measure. Interestingly, acute kidney injury did not occur despite very high CPK levels. With phosphate correction the rhabdomyolysis gradually responded with a decline in CPK levels and subsequent normalization. The patient was fully recovered by day 10 and discharged home with normal CPK.

This case report highlights the possibility of rhabdomyolysis as an uncommon complication of severe falciparum malaria as well as hypophosphatemia as a possible etiology for the problem.

Conflict of Interest: Authors declare no conflict of interest

Ethics: Informed and written consent was obtained from the patient

\section{Reference:}

1. WHO certifies Sri Lanka malaria-free, SEAR/PR/1631. September 05, 2016. http://www.searo.who.int/mediacentre/releases/2016/1631/en/ accessed on Sep 07, 2016.

2. De Silva HJ, Goonetilleke AKE, Senaratna N, et al. Skeletal muscle necrosis in severe falciparum malaria. Br Med J. 1988, 296:1039 doi: http://doi.org/10.1136/bmj.296.6628.1039.

3. Sinniah R, Lye W. Acute renal failure from myoglobinuria secondary to myositis from severe falciparum malaria. Am $J$ Nephrol. 2000; 20:339-343. doi: http://doi.org/10.1159/000013611

4. Miller KD, White NJ, Lott JA, et al. Biochemical evidence of muscle injury in African children with severe malaria. J Infect Dis 1989; 159:139-142. 
doi: http://dx.doi.org/10.1093/infdis/159.1.139

5. Flores EA, Bistrian BR, Pomposelli JJ, et al. Infusion of tumor necrosis factor/cachectin promotes muscle catabolism in the rat: a synergistic effect with interleukin 1. J Clin Invest 1989; 83:1614-1622.

doi: http://doi.org/10.1172/JCI114059

6. Haber R and Browner W. Fever as a cause of hypophosphatemia in patients with malaria. PLoS One. 2007; 2(12):e1380.

doi: http://doi.org/10.1371/journal.pone.001380 